\title{
Optimization of Desiccant Absorption System Using a Genetic Algorithm
}

\author{
Ayman A. Aly ${ }^{1,2}$ \\ ${ }^{1}$ Mechatronics Section, Mechanical Engineering Department, Faculty of Engineering, Taif University, Al-Haweiah, Saudi Arabia; \\ ${ }^{2}$ Mechatronics Section, Mechanical Engineering Department, Faculty of Engineering, Assiut University, Assiut, Egypt. \\ Email: draymanelnaggar@yahoo.com
}

Received June 30 ${ }^{\text {th }}$, 2011; revised July 25 ${ }^{\text {th }}$ 2011; accepted August $3^{\text {rd }}, 2011$.

\begin{abstract}
Optimization of the open absorption desiccant cooling system has been carried out in the present work. A finite difference method is used to simulate the combined heat and mass transfer processes that occur in the liquid desiccant regenerator which uses calcium chloride $\left(\mathrm{CaCl}_{2}\right)$ solution as the working desiccant. The source of input heat is assumed to be the total radiation incident on a tilted surface. The system of equations is solved using the Matlab-Simulink platform. The effect of the important parameters, namely the regenerator length, desiccant solution flow rate and concentration, and air flow rates, on the performance of the system is investigated. In order to optimize the system performance, a genetic algorithm technique has been applied. The system coefficient of performance COP has been maximized for different design parameters. It has been found that the maximum values of COP could be obtained for different combinations of regenerator length solution flow rate and air flow rate. Therefore, it is essential to select the design parameters for each ambient condition to maximize the performance of the system.
\end{abstract}

Keywords: Genetic Algorithm, Modeling, Optimization, Solar-Powered, Desiccant

\section{Introduction}

In hot and humid areas, liquid desiccant air-conditioning systems have been proposed as alternatives to the conventional vapor compression cooling systems to control air humidity. Since the introduction of open-cycle liquiddesiccant absorption solar-cooling system by Kakabaev and Khandurdyev [1], the system has been investigated extensively. The feasibility of the system and the advantages it can offer in terms of energy and cost saving have been proved in different climates (see for example [2-6]). Among the advantages of liquid-desiccant systems are: the lower temperature for regeneration, the ease of manipulation, lower pressure drop in the contactors with the flowing air, and the possibility of filtering to remove dirt taken in from the air [7].

A schematic of an open solar absorption cooling system is shown in Figure 1. The weak absorbent solution is heated and subsequently concentrated in the solar collector. The strong regenerated solution leaves the collector and passes through a liquid column, to allow the strong solution to go from atmospheric pressure to reduced pressure efficiently. The strong solution then passes through a regenerative heat exchanger on its way to the absorber, where it absorbs water from the evaporator, maintaining the reduced pressure required with the energy supplied by heat from the cold space. The resultant weak solution is pumped from the absorber back to atmospheric pressure through the regenerative heat exchanger and the collector, completing the cycle. The advantages of this system would include a simpler collector, which also acts as a regenerator, and a reduction in thermal losses. The overall performance of the system is governed entirely by the rate at which water is driven from the solution in the collector, since this determines the flow of water that can be introduced into the evaporator as refrigerant. The rate of water evaporation from the regenerator gives a direct measure of the system cooling capacity.

A simplified analytical procedure for calculating the mass of water evaporated from the weak solution in the solar regenerator in terms of climatic conditions and solution properties at the regenerator inlet has been developed by Kakabayev and Khandurdyev [1]. Yang and Wang performed a computer simulation for the collector/ regenerator using a radiation processor which makes use of the statistical meteorological data for the summer season at Kaohsiung, Taiwan [8]. Alizadeh and Saman developed a computer model to study the thermal perfor- 


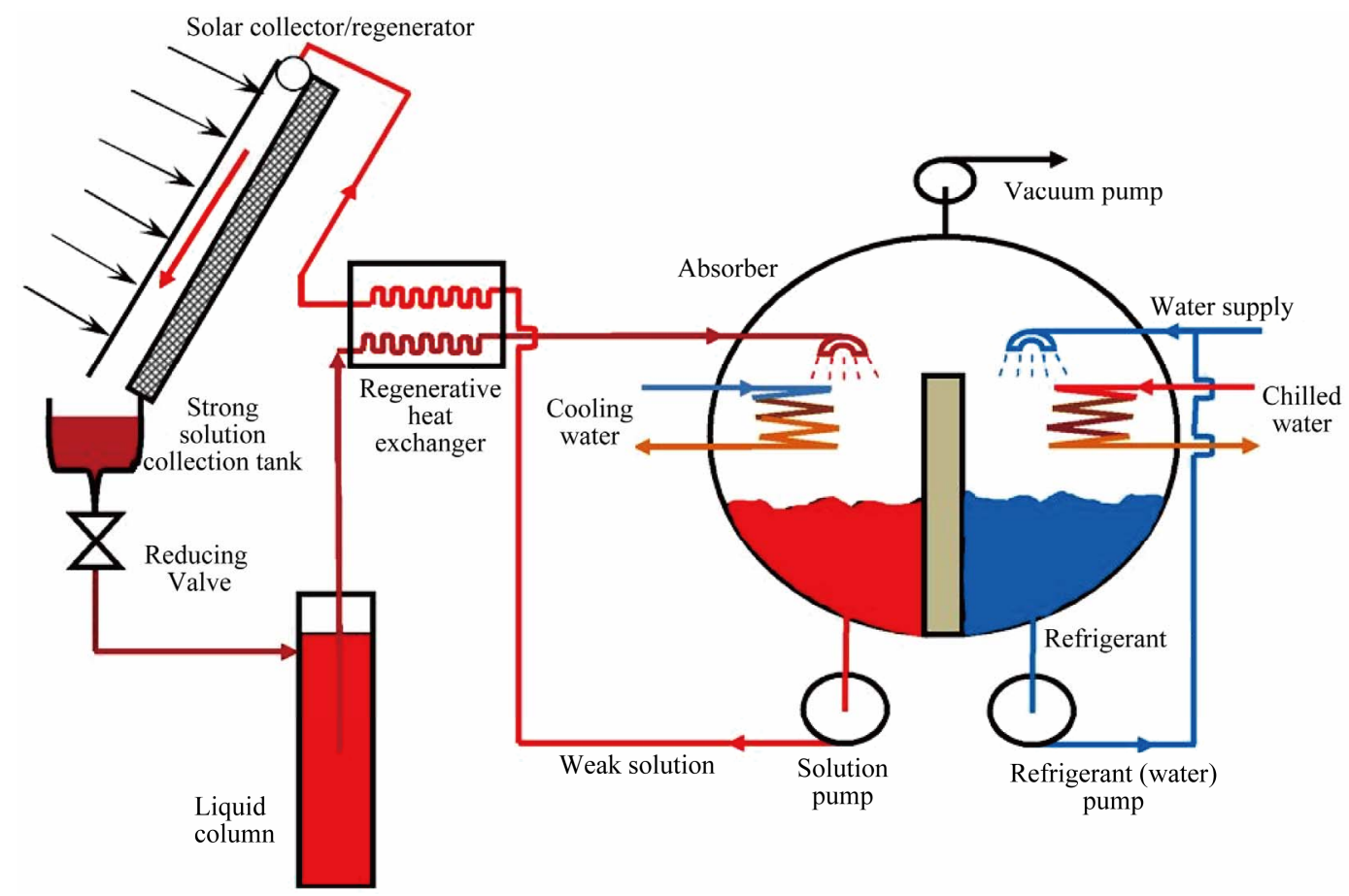

Figure 1. Schematic of the solar-powered open absorption cooling system.

mance of a forced parallel flow solar regenerator [9]. A parametric analysis of the system has been performed to calculate the rate of evaporation of water from the solution as a function of the system variables and the climatic conditions. However, the solar radiation intensity was assumed constant in the analysis. More recently, an artificial neural network model has been used with a finite difference method to simulate the combined heat and mass transfer processes that occur in the liquid desiccant regenerator [10].

To maximize the economic benefits of the solarpowered desiccant cooling systems, it is necessary to optimize the performance of the absorption desiccant cooling core. Genetic algorithms (GAs) have been recognized as effective techniques to solve optimization problems. A GA starts with a population of randomly generated populations, and advances towards better populations by applying genetic operators modeled on the genetic processes occurring in nature. Compared with other optimization techniques; a GA is superior in avoiding local minima which is a common aspect in nonlinear systems [11]. The objective of this work is to present a method for the optimization of solar-powered open absorption desiccant cooling system using a GA.

\section{System Model}

\subsection{Collector/Regenerator (C/R) Model}

The C/R shown in Figure 2 employs an inclined flat blackened surface over which the absorbent solution to be concentrated trickles down as a thin liquid film. The channel is divided into a large number of equal segments of width $d x$ with the assumption of constant properties within the segment (air vapour pressure, $p_{a}$, and temperature, $T_{a}$, and vapor pressure on the solution surface, $p_{s}$, temperature, $T_{s}$, and concentration, $C_{s}$ ). The main equations include the energy balance and mass balance for each segment of the open-cycle regenerator. These equations are summarized as follows [10]:

Energy balance for the regenerator-segment:

$$
I_{a} d x=m_{s} d H_{s}+m_{a} d H_{a}+U_{L}\left(T_{s}-T_{0}\right)+m h_{f g}
$$

where $m_{a}$, and $m_{s}$ are the mass flow rates of air and solution $\mathrm{kg} / \mathrm{s}$, respectively, $H_{a}$, and $H_{s}$ are the specific enthalpies of air and solution $\mathrm{J} / \mathrm{kg}$, respectively, $m$ is the mass of evaporated water, $\mathrm{kg} / \mathrm{s}, h_{f g}$ is latent heat of water, $\mathrm{J} / \mathrm{kg}, T_{0}$ is the outside temperature, and $U_{L}$ is the overall heat loss coefficient, $\mathrm{W} / \mathrm{m}^{2}{ }^{\circ} \mathrm{C}$.

Energy balance for the air stream passing through the regenerator-segment:

$$
m_{a} d H_{a}=h_{a}\left(T_{s}-T_{a}\right) d x-h_{s}\left(T_{a}-T_{0}\right) d x
$$

where $h_{a}$ and $h_{s}$ are heat transfer coefficients for the air and solution sides, each in $\mathrm{W} / \mathrm{m}^{2}{ }^{\circ} \mathrm{C}$.

The amount of water evaporated from the weak solution: 


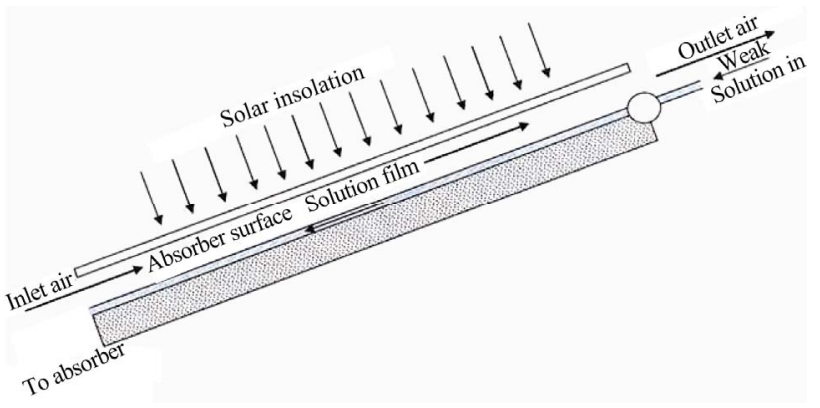

Figure 2. Open-cycle solar collector regenerator.

$$
m=0.622 \frac{m_{a}}{p_{b}}\left(p_{a}-p_{a i}\right)
$$

where $p_{b}$ and $p_{a i}$ are the barometric pressure and initial vapour pressure in air at the regenerator inlet, in $\mathrm{mm} \mathrm{Hg}$, respectively.

The rate of mass transport of water vapor:

$$
\frac{d m}{d x}=\beta\left(p_{s}-p_{a}\right)
$$

where $\beta$ is the mass transfer coefficient, $\mathrm{kg} / \mathrm{s} \mathrm{m}^{2} \mathrm{mmHg}$. And the relation between the mass of evaporated water and solution flow rates is given by:

$$
C_{s}=C_{s i} /\left(1-\frac{m}{m_{s}}\right)
$$

where $C_{s i}$ is the initial concentration of the solution at regenerator inlet.

The relationship between the solution temperature, concentration and vapor pressure for calcium chloride $\left(\mathrm{CaCl}_{2}\right)$ is given by,

$$
p_{s}=a+b T_{s}+\frac{c}{C_{s}}
$$

where $a, b$, and $c$ are empirical constants [9].

\section{System coefficient of performance (COP)}

The overall coefficient of performance of the system can be evaluated from the following expression:

$$
C O P=\frac{Q}{I_{t} A_{c}}
$$

where $A_{c}$ is the collector area and $Q$ is the cooling rate of the desiccant cooling system, which can be evaluated by multiplying the rate of water evaporation, $m$ by the latent heat of water $L$ at the evaporator pressure i.e.,

$$
Q=m L
$$

The above mentioned analysis shows the dependence of the regeneration process on operational parameters such as air and liquid mass flow rates as well as the vapour pressure of inlet air. In this study, the performance of soft computing methodology, is used for the system performance analysis.

\subsection{Simulation Procedure}

\subsubsection{Solving the Equations}

The theoretical model forms a system of coupled nonlinear ordinary differential equations and algebraic equations which link the characteristic parameters of air and desiccant solution. An analytical solution is rather difficult and could only be obtained for simplified situations that allow the reduction of the basic equations. In the current study, a numerical solution is obtained by the finite difference technique. These equations are solved using the Matlab-Simulink platform that allows the system to be modeled by drawing a block diagram directly on the screen. The Simulink representation of the system of equations is presented in Figure 3. Each block represent a calculation unit and may be composed of more detailed sub-systems. The sub-system for calculating the mass of evaporated water is shown in Figure 4. A Matlab computer code is written to perform the computations and visualize the results. The ordinary differential equations are solved using the fourth-order Runge-Kutta scheme with variable time steps.

\subsubsection{Collector/Regenerator Module}

The system of equations from (1) to (5) has 6 unknowns; which are: $m, T_{a}, T_{s}, p_{a}, p_{s}$ and $C_{s}$. Given the input values of mass flow rates of air and solution, air temperature, and solution concentration at the inlet of any segment, along with the physical properties of the working desiccant, the output values are obtained using the above equations by a step-by-step analysis up to the outlet. An iterative procedure is used to obtain a numerical solution with the set accuracy criterion. The heat and mass transfer coefficients are evaluated by using available correlations from the literature [12].

\subsubsection{Optimization Technique}

The traditional method for finding the optimum solution of system parameters is to optimize the system model for

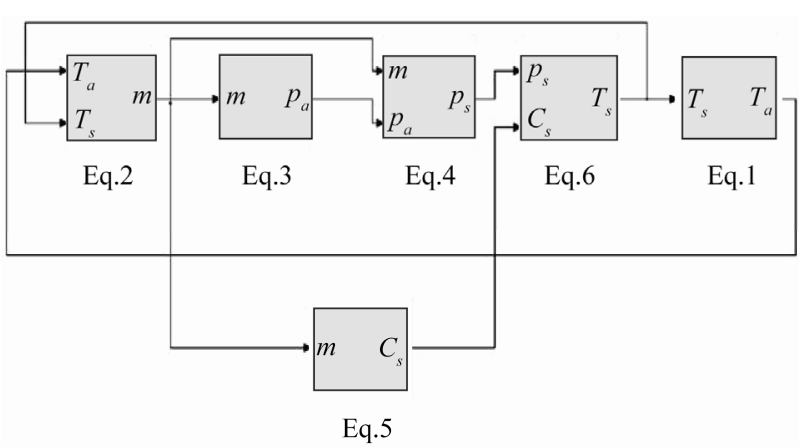

Figure 3. Scheme of the Matlab-Simulink program. 


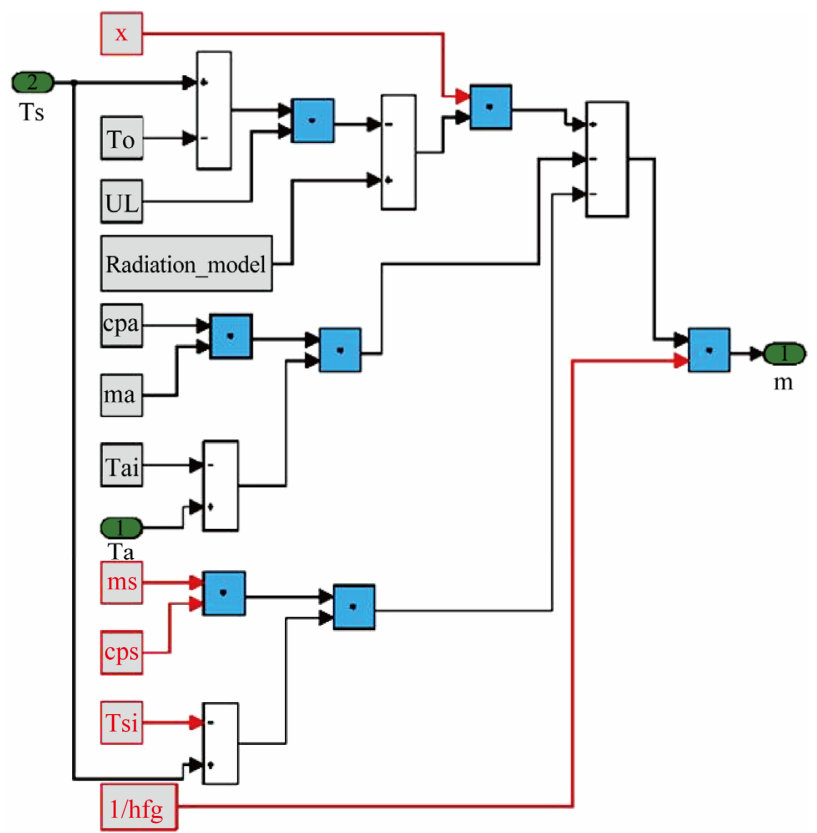

Figure 4. Sub-block for calculating the mass of evaporated water.

each parameter and decide the characteristics of the system which give local optimum solution. This method may lead to solutions far away from the optimum as the method strongly depends on the peculiarities of the system and the intuition of the modeler. In addition, each run might need several minutes to hours to be performed depending on the computer system frequency and the complexity of the system. Thus, it is required to be able to find the optimum solution for the system under consideration as well as to reduce the time required for each task to be performed. A different approach to optimize the system based on a genetic algorithm (GA) technique is suggested in this paper to find the optimum values of regenerator length, solutions flow rate and air flow rate mass, which will maximize the total collected water from the system and consequently the coefficient of performance. The genetic algorithm is briefly described in this section.

GA starts with an initial population containing a number of chromosomes where each one represents a solution of the problem of choosing the designed model parameters which performance is evaluated by a fitness function of the tested model as represented in the flow chart in Figure 5. The solution expression of the bit code is decoded to values used in an application task. This is a phenotype expression.

Genetic programming breeds computer programs by executing the following three steps:

1) Generate an initial population of compositions of the functions (regenerator length, solution flow rate and

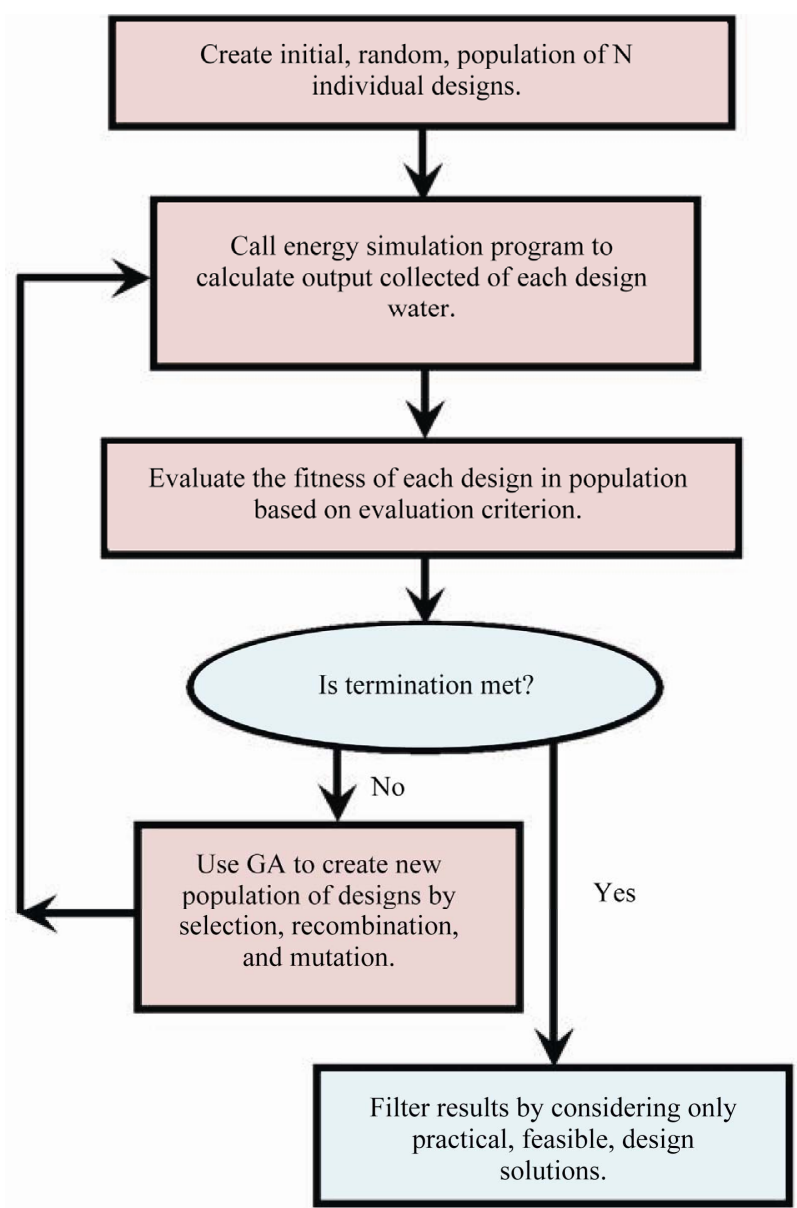

Figure 5. Flow chart of the optimization tool.

air flow rate).

2) Iteratively perform the following sub-steps (referred to herein as a generation) on the population of programs until the termination criterion has been satisfied:

a) Execute each program in the population and assign a fitness value using the fitness measure which maximize of the evaporated mass of water.

b) Create a new population of programs by applying the following operations. The operations are applied to program selected from the population with a probability based on fitness (with reselection allowed).

i) Reproduction: Copy the selected program to the new population. The reproduction process can be subdivided into two sub-processes: Fitness Evaluation and Selection. The fitness function is what drives the evolutionary process and its purpose is to determine how well a string (individual) solves the problem, allowing for the assessment of the relative performance of each population member.

ii) Crossover: Create a new offspring program for the new population by recombining randomly chosen parts of two selected programs. Reproduction may proceed in three steps as follows: a) two newly reproduced strings 
are randomly selected from a Mating Pool, b) a number of crossover positions along each string are uniformly selected at random, and c) two new strings are created and copied to the next generation by swapping string characters between the crossovers positions defined before.

iii) Mutation: Create one new offspring program for the new population by randomly mutating a randomly chosen part of the selected program.

iv) Architecture-altering operations: Select an architecture-altering operation from the available repertoire of such operations and create one new offspring program for the new population by applying the selected architecture-altering operation to the selected program.

3) Designate the individual program that is identified by result designation (e.g., the best so-far individual) as the result of the run of genetic programming. This result may be a solution (or an approximate solution) to the problem.

The design specifications of the GA are shown in Table 1.

For more details of genetic operators and each block in the flowchart, one may consult literature $[13,14]$.

Here the goal is to find sets of system parameters that will give a minimum fitness value over the operating period $[0, t]$. The GA initializes a random set of population of these three variables (regenerator length, solutions flow rate and air flow rate mass).

Main system calculation parameters are presented in Table 2. It should be noted that, moderate values of the ambient parameters (temperature and humidity) are selected for simulation purposes. However, variation of the desiccant initial concentration may affect the value of the system coefficient of performance (COP) but the optimum design parameters will be the same values obtained at the specified concentration which is used in the optimization process.

Table 1. Specification of the GA.

\begin{tabular}{lc}
\hline Population size & 20 \\
Individuals in offspring generation & 50 \\
Coding of individuals & Gray-coding \\
Recombination probability & 0.6 \\
Crossover rate & 0.7 \\
Mutation rate & 0.05 \\
Chromosome length & 12 \\
Precision of variables & 3 \\
Generation gap & 1 \\
\hline
\end{tabular}

Table 2. Calculation parameters of the system.

\begin{tabular}{ll}
\hline Ambient temperature, ${ }^{\circ} \mathrm{C}$ & 33 \\
Ambient vapor pressure, mm Hg & 20 \\
Desiccant $\left(\mathrm{CaCl}_{2}\right)$ initial concentration, \% & 40 \\
Radiation intensity, $\mathrm{kW} / \mathrm{m}^{2}$ & 0.8 \\
\hline
\end{tabular}

\section{Results and Discussion}

The performance of the solar collector/regenerator is influenced by design parameters (regenerator length, solution flow rate, working solution concentration and air flow rate) and ambient conditions (air temperature and vapor pressure in the flowing air). These key parameters are investigated in the following sub-sections. A sensitivity analysis is performed by varying the parameters of interest one at a time, while keeping all others fixed at given values.

In order to analyze the effect of air mass flow rate on the regeneration process, the solution mass flow rate, $m_{s}$, is settled at $20 \mathrm{~kg} / \mathrm{hr}$ and the range of air mass flow rate, $m_{a}$, is considered in the range $(10 \mathrm{~kg} / \mathrm{hr}-200$ $\mathrm{kg} / \mathrm{hr}$ ), then the vapour pressure difference between the regenerated solution and flowing air is plotted versus the regenerator length. For a given regenerator length, the vapour pressure, which is the mass transfer potential, is directly proportional with the rate of water evaporation, when the mass transfer coefficient is assumed constant. As shown in Figures 6 and 7, the vapor pressure difference has a maximum for a given length of the regenerator. The length, at which the maximum rate of evaporation occurs, increases with the air flow rate. Concerning the effect of solution inlet concentration on regeneration process, the decrease of solution concentration can effectively improve the regenerator performance, though it sacrifices solution outlet concentration.

The coefficient of performance (COP) of the system is illustrated in the surface plot shown in Figure 8. For the specified operating conditions, a maximum value of the COP occurs at a given range of air and solution flow rates. However, the maximum value of COP is dependent of the design parameters and operating conditions, therefore it is essential to select the design parameters for each ambient condition to maximize the COP of the system.

Table 3 demonstrates the simulation results for the maximum values of the system COP, when the genetic algorithm is applied. It can be observed that the maximum values of COP range from $32.5 \%$ to $36.6 \%$. However, for the three design parameters, optimum values of COP could be attained for different combinations of input parameters. Comparing the GA outputs presented in Table 3 with the simulation results plotted in Figure 8, it can be found the optimum values of COP obtained from the GA are in good agreement with the maximum values presented in Figure 8. Moreover, it should be noted that the application of genetic algorithm results in direct evaluation of the optimization parameters. When designing an optimal system, multiple options are available and the decision must be taken on the account of the availability of the site and the economical considerations. 


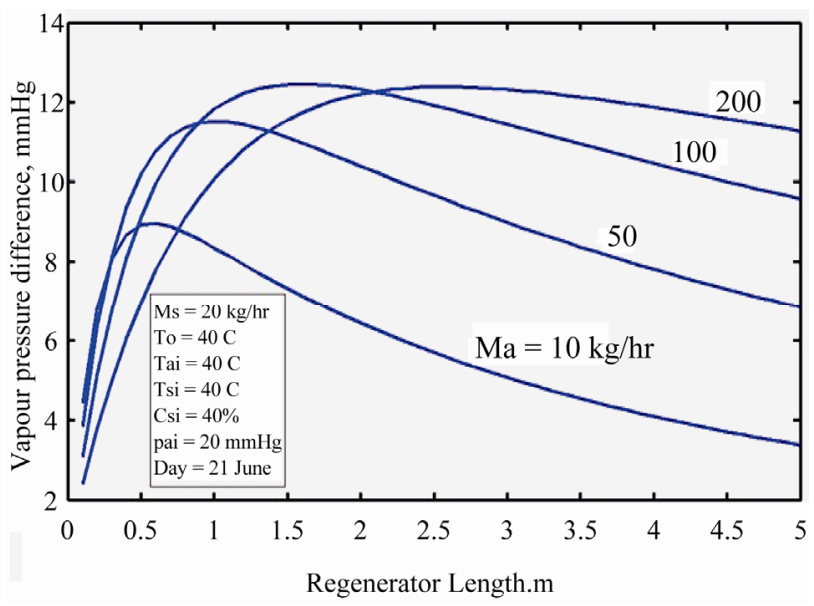

Figure 6. Variation of vapor pressure at regenerator exit with different values of air flow rate, at noon time.

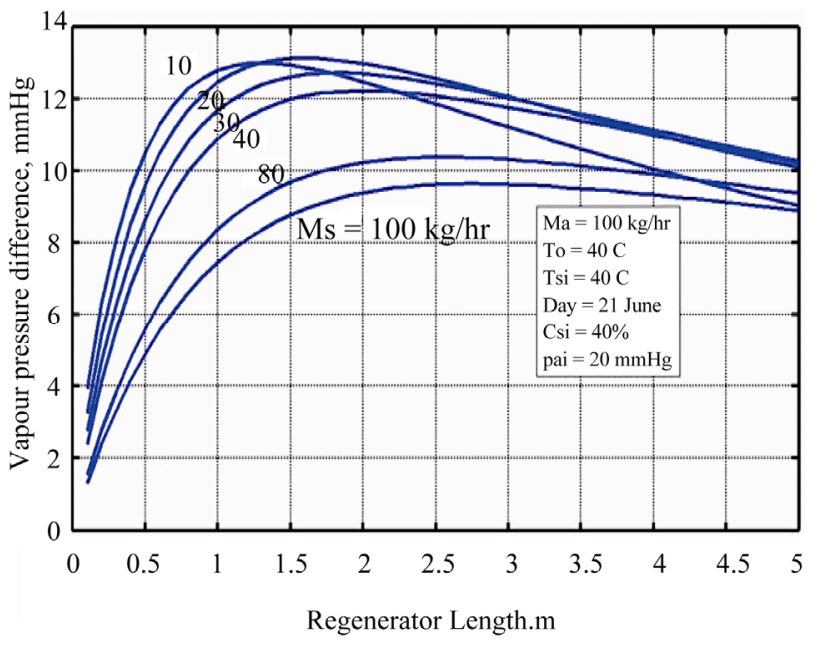

Figure 7. Variation of vapor pressure at the regenerator exit for different values of solution flow rate, at noon time.

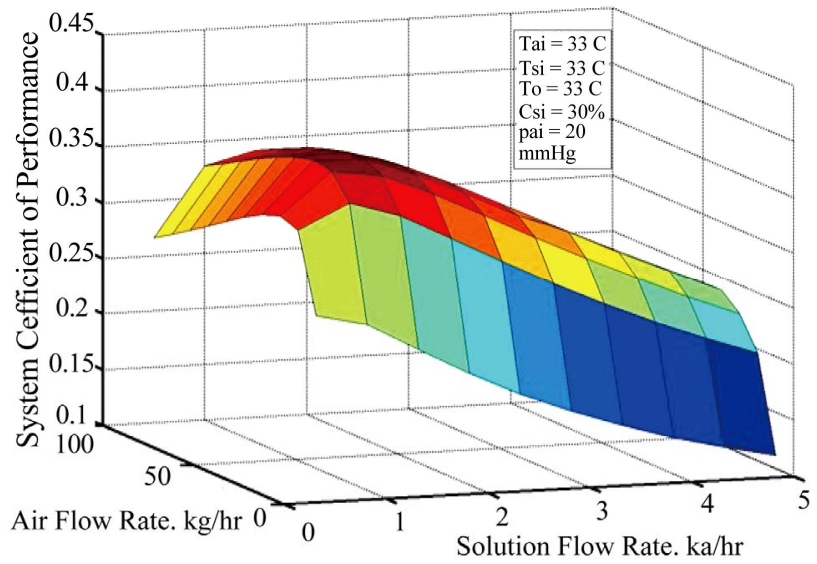

Figure 8. Surface plot showing the variation of system COP with air and solution flow rates.
Table 3. System optimization parameters produced by GA.

\begin{tabular}{cccc}
\hline $\begin{array}{c}\text { Overall coefficient } \\
\text { of performance, \% }\end{array}$ & $\begin{array}{c}\text { Air flow } \\
\text { rate, } \mathrm{kg} / \mathrm{hr}\end{array}$ & $\begin{array}{c}\text { Desiccant flow } \\
\text { rate, } \mathrm{kg} / \mathrm{hr}\end{array}$ & $\begin{array}{c}\text { Collector } \\
\text { length, } \mathrm{m}\end{array}$ \\
\hline 33.3 & 21.96 & 0.76 & 2.7546 \\
36.2 & 19.44 & 0.95 & 6.5665 \\
33.3 & 17.28 & 0.71 & 1.9707 \\
36.6 & 17.64 & 0.97 & 9.2308 \\
35.3 & 10.08 & 0.95 & 3.4505 \\
36.5 & 13.68 & 0.97 & 6.18156 \\
36.5 & 18.72 & 0.97 & 2.7766 \\
32.5 & 35.28 & 0.94 & 7.2796 \\
34.0 & 13.68 & 0.74 & 3.3919 \\
\hline
\end{tabular}

\section{Conclusions}

The optimization of solar-powered desiccant regenerator used for open absorption cooling cycle is presented. A finite difference method is used to simulate the combined heat and mass transfer processes that occur in the liquid desiccant regenerator. The model is implemented using the Matlab-Simulink platform along with a genetic algorithm in order to optimize the system performance. It is concluded that the proposed model can be successfully used for predicting and optimizing the overall performance of the system. The simulation has shown that the vapor pressure difference has a maximum value for a given regenerator length. It is also shown that for specified operating conditions, a maximum value of the coefficient of performance occurs at given values of air and solution flow rates and regenerators length. Therefore, it is essential to select the design parameters for each ambient condition to maximize the coefficient of performance of the system.

\section{REFERENCES}

[1] A. Kakabaev and A. Khandurdyev, "Absorption Solar Refrigeration Unit with Open Regeneration of Solution,” Gliotekhnika, Vol. 5, No. 4, 1969, pp. 28-32.

[2] R. Yang and P. L. Wang, "Experimental Study of a Forced Convection Solar Collector/Regenerator for Open Cycle Absorption Cooling," Transactions of the ASME Journal of Solar Energy Engineering, Vol. 116, 1994, pp. 194-199. doi:10.1115/1.2930081

[3] G. Grossman, "Solar-Powered Systems for Cooling, Dehumidification and Air-Conditioning," Solar Energy, Vol. 72, No. 1, 2002, pp. 53-62. doi:10.1016/S0038-092X(01)00090-1

[4] M. Krause, W. Saman and K. Vajen, “Open Cycle Liquid Desiccant Air Conditioning Systems-Theoretical and Experimental Investigations,” ANZSES Conference, Dunedin, New Zealand, 2005.

[5] K. Daou, R. Z. Wang and Z. Z. Xia, "Desiccant Cooling Air Conditioning: A Review,” Renewable and Sustainable Energy Reviews, Vol. 10, 2006, pp. 55-77. doi:10.1016/j.rser.2004.09.010

[6] J. Dieckmann, K. Roth and J. Brodrick, "Liquid Desic- 
cant Air Conditioners,” ASHRAE Journal, Vol. 50, No. 10, 2004, pp. 90-95.

[7] H. Factor and G. A. Grossman, "A Packed Bed Dehumidifier/Regenerator for Solar Air Conditioning with Liquid Desiccant," Solar Energy, Vol. 24, 1980, pp. 541-550. doi:10.1016/0038-092X(80)90353-9

[8] R. Yang and P. L. Wang, "A Simulation Study of the Performance Evaluation of Single-Glazed and DoubleGlazed Collectors/Regenerators for an Open-Cycle Absorption Solar Cooling System,” Solar Energy, Vol. 71, No. 4, 2001, pp. 263-268. doi:10.1016/S0038-092X(01)00047-0

[9] S. Alizadeh and W. Saman, "Modeling and Performance of a Forced Flow Solar Collector/Regenerator Using Liquid Desiccant," Solar Energy, Vol. 72, No. 2, 2002, pp. 143-154. doi:10.1016/S0038-092X(01)00087-1

[10] A. A. Aly, E. B. Zeidan and A. M. Hamed, "Performance

\section{Nomenclature}

$A_{c}$
$a, b$, and $c$
$h$
$I$
$m$
$p$
$Q$
$T$
$U_{L}$

collector surface area, $\mathrm{m}^{2}$ empirical constants (Equation (17)) heat transfer coefficient, $\mathrm{W} / \mathrm{m}^{2}{ }^{\circ} \mathrm{C}$ solar radiation intensity, $\mathrm{W} / \mathrm{m}^{2}$ rate of water evaporation, $\mathrm{kg} / \mathrm{s}$ vapor pressure, mmHg cooling rate, $\mathrm{W}$ temperature, ${ }^{\circ} \mathrm{C}$ overall heat loss coefficient, $\mathrm{W} / \mathrm{m}^{2}{ }^{\circ} \mathrm{C}$

\section{Greek Symbols}

$\begin{array}{ll}\beta & \text { mass transfer coefficient, } \mathrm{kg} / \mathrm{s} . \mathrm{m}^{2} \mathrm{mmHg} \\ \tau & \text { transmittance }\end{array}$

Evaluation of Open-Cycle Solar Regenerator Using Artificial Neural Network Technique,” Journal of Energy and Buildings, Vol. 43, 2011, pp. 454-457.

[11] J. R. Koza, M. A. Keane, M. J. Streeter, W. Mydlowec, J. $\mathrm{Yu}$ and G. Lanza, "Genetic Programming IV: Routine Human-Competitive Machine Intelligence,” Kluwer Academic Publishers, Boston, 2003.

[12] W. L. McCabe, J. C. Smith and P. Harriott, "Unit Operation of Chemical Engineering,” McGraw-Hill, New York, 1985.

[13] C. R. Reeves, "Using Genetic Algorithms with Small Populations," Proceedings of the 5th International Conference on Genetic Algorithms, 1993, pp. 92-99.

[14] D. E. Goldberg, "Genetic Algorithms in Search, Optimization and Machine Learning,” Addison-Wesley, Boston, 1989.

$\begin{array}{ll}\text { Subscripts } & \\ a & \text { air and absorbed } \\ C & \text { collector } \\ i & \text { inlet } \\ L & \text { liquid } \\ O & \text { outside } \\ \text { max } & \text { maximum } \\ S & \text { solution and surface } \\ T & \text { total and tilted }\end{array}$

